\title{
Alexithymia and Interleukin Variations in Somatoform Disorder
}

\author{
Francisco Pedrosa Gil ${ }^{a}$ Marius Nickel ${ }^{d,}$ e Nathan Ridout ${ }^{f} \quad$ Markus J. Schwarz $^{c}$ \\ Claudia Schoechlin ${ }^{c}$ Ralf Schmidmaier ${ }^{b}$

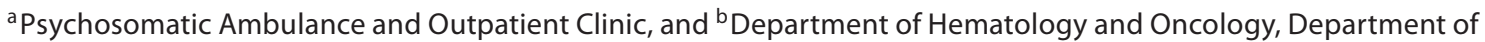 \\ Internal Medicine Innenstadt, and ' Psychiatric and Psychotherapeutic Hospital, Ludwig Maximilians University, \\ Munich, Germany; ${ }^{d}$ University Clinic for Psychosomatics and Psychotherapy, Medical University Graz, Bad Aussee, and \\ eUniversity Clinic for Psychiatry 1, PMU, Salzburg, Austria; ${ }^{\mathrm{f} C l i n i c a l ~ a n d ~ C o g n i t i v e ~ N e u r o s c i e n c e s ~ R e s e a r c h ~ G r o u p, ~}$ \\ School of Life and Health Sciences, Aston University, Birmingham, UK
}

\section{Key Words \\ Somatoform disorders · Interleukin · Alexithymia}

\begin{abstract}
Objective: The aim of the present study was to investigate if somatoform disorders (SFD) are associated with changes in the normal serum levels of important interleukins, and further, to establish if these changes are related to the presence and severity of alexithymia in patients with SFD. Methods: Twenty-four unmedicated patients who met the International Classification of Diseases (ICD-10) diagnostic criteria for SFD completed the psychological questionnaire to assess alexithymia (Toronto Alexithymia Scale), symptom reporting (SCL-90-R) and diagnostic criteria for SFD (Screening for Somatoform Symptoms scale). Serum concentrations of soluble interleukin 2 receptor $\alpha$ (sIL-2 R $\alpha$ ), IL-4, IL-6, IL-10 and IL-12 were determined in patients with SFD and in 9 healthy subjects. Results: In patients with SFD, serum levels of IL-6 ( $p<$ $0.001), I L-10(p=0.047)$ and immunoglobulin $E(p=0.045)$ were significantly increased in comparison with healthy controls. Additionally, a negative correlation was observed between the level of alexithymia ('total' Toronto Alexithymia Scale score) and the serum levels of sIL-2 R $\alpha(r=-0.538)$ in SFD. Conclusions: Taken together, these results suggest that SFD, with clinically significant alexithymia, are associated
\end{abstract}

\section{KARGER \\ Fax +41613061234 \\ E-Mail karger@karger.ch}

www.karger.com (c) 2007 S. Karger AG, Basel 1021-7401/07/0145-0235\$23.50/0

Accessible online at:

www.karger.com/nim with a reduction in Th1-mediated immune function and an increase in the activation of the Th2 immune function, indicated by the augmented serum levels of IL- 6 and IL-10 and elevated immunoglobulin E. Copyright $\odot 2007$ S. Karger AG, Basel

\section{Introduction}

In 1980, the Diagnostic and Statistical Manual of Mental Disorders (DSM-III) introduced the category of somatoform disorders (SFD) as a provisional grouping of psychiatric conditions characterized by often multiple and variable somatic symptoms - commonly seen in general medical practice and primary care - but defying medical explanation [1]. It is clear from their high lifetime prevalence $(4-5 \%)$ in the general population [2,3], their high comorbidity with other conditions, their debilitating effect on patients, and their association with high levels of drug use, that SFD are of great clinical and economic importance [4].

Previous studies [e.g., ref. 5, 6] have reported that, relative to healthy participants, patients with SFD exhibit elevated levels of alexithymia. Alexithymia is a concept that was developed by Sifneos [7] and is characterized by an inability to describe and identify feelings, by an ab-

Francisco Pedrosa Gil, MD, Psychosomatic Outpatient Clinic Department of Internal Medicine, Ludwig Maximilians University Pettenkoferstrasse 10, DE-80336 Munich (Germany) Tel. +49895160 3572, Fax +498951604751

E-Mail Francisco.Pedrosa.Gil@med.uni-muenchen.de 
sence of fantasies, and by the tendency to utilize an externally focused analytical cognitive style. The concept of alexithymia has been examined in a variety of different medical, psychosomatic and psychiatric disorders $[8,9]$ and supports the clinical impression of an association between somatization and alexithymia $[6,10]$

It is notable that previous studies [e.g., ref. 11] have reported that certain cytokines can stimulate inflammatory pain. It would seem plausible that the inflammatory response of the immune system (mediated by cytokines) might underlie the illness symptoms reported by patients with SFD. However, contrary to this notion, Rief et al. [12] reported that patients with somatization syndrome actually exhibited changes in immune function that are associated with a moderate reduction in the inflammatory response, such as reduced levels of proinflammatory cytokines such as interleukin (IL)-6 and lowered CD8 (lowered T-lymphocytic activity), and yet, on the other hand, increased IL-1 receptor antagonist with monocytic activation. This apparent inconsistency is worth of further study.

As noted previously, alexithymia is related to an increase in the bodily expression of emotional distress. With this in mind, it is plausible that high levels of alexithymia would be associated with changes in immune function. Todarello et al. $[13,14]$ reported lower counts of almost all lymphocyte subsets in alexithymic patients compared with non-alexithymic patients. Similarly, Dewaraja et al. [15] reported that healthy men categorized as highly alexithymic exhibited decreased cytotoxic lymphocyte counts (for natural killer subset CD57CD16+ cells and killer effective T cell CD8+CD11a+ cells). In a recent study, Corcos et al. [16] examined alexithymia, mood (depression and anxiety) and serum levels of the different cytokines in a group of 17 healthy women and found a positive correlation between the participants' scores on the Toronto Alexithymia Scale (TAS) and their serum levels of IL-4. Moreover, Kelly-Welch et al. [17] reported that the deregulation of anti-inflammatory cytokines such as IL-4 may lead to a disturbance of the type 1/type 2 cytokine balance. A major consequence of this cytokine imbalance is an increase in Th2 response, with a resultant increase in immunoglobulin $\mathrm{E}$ ( $\mathrm{IgE}$ ) antibody synthesis leading to an inflammatory response in the body.

As there have only been a few studies examining activation of the inflammatory response system and changes in immunological variables in SFD $[17,18]$, and similarly, as there has been relatively little research addressing the effects of alexithymia on the immune function [e.g., ref.
$16,19]$, it is important to extend this research in order to develop our understanding of the role of the immune response in SFD. Considering that patients with SFD have been shown to exhibit elevated levels of alexithymia $[5,6]$, it is plausible that the observed changes in the immune function of patients with SFD might relate to differences in concomitant alexithymia. However, as yet, there has been no study that has examined the relationship between alexithymia and cytokine production in patients with SFD. Therefore, the aim of the present study was to conduct such an investigation.

In the present study, serum levels of key interleukins, i.e. IL-4, IL-6, IL-10, IL-12 and soluble IL-2 receptor $\alpha$ (sIL-2 R $\alpha$ ), were systematically examined in a group of patients with SFD and a group of healthy controls. The presence and severity of alexithymia in patients with SFD was also established using the TAS. In line with previous work, we predicted a higher prevalence of alexithymia in SFD compared with the population norms [20, 21]. Second, it was predicted that there would be changes in the serum levels of the key cytokines in the patients with SFD relative to the healthy controls. Furthermore, it was expected that these changes in serum levels would be significantly correlated with the patients' alexithymia (TAS26) scores.

\section{Methods}

\section{Participants}

Twenty-four patients (16 females, 8 males) meeting the International Classification of Diseases (ICD-10) criteria for SFD and 9 healthy controls (4 females, 5 males) took part in the present study. Ten of the SFD patients were diagnosed with somatization disorder (F45.0), 13 with somatoform autonomic dysfunction (F45.3), and 1 with persistent somatoform pain disorder (F45.4). The characteristics of the patients are presented in table 1 . The patients with SFD were recruited from an outpatient clinic of the Department of Medicine (Munich University), where they had been referred for a diagnostic interview and counselling in the psychiatric and psychosomatic field. Inclusion criteria for the patient group were the presence of SFD diagnosed according to the ICD-10 criteria. The diagnosis was established through a clinical interview conducted by a trained psychiatrist (P.G.F.), who performed extensive physical and psychological assessment of the patients, including checking for the signs and symptoms of SFD as outlined in the diagnostic criteria cited in the ICD-10 [22]. A further aid to the diagnosis of SFD was that the participants fulfill the criteria on the Screening for Somatoform Symptoms (SOMS) questionnaire [23]. Physical conditions (e.g., angina) that may have explained the patients' symptoms had been excluded prior to referral following extensive inpatient or outpatient investigation at the Department of Medicine or in general practice. The presence of an acute or chronic infection or a recent history of an 
infectious disease (e.g., influenza) within the past 4 weeks was a further exclusion criterion. Exclusion criteria for the patient group were also the presence of medical disorders (e.g., neoplasms, autoimmune, cardiac, pulmonary or endocrine diseases), severe mental illnesses, such as schizophrenia, schizoaffective disorder, bipolar disorder, substance abuse disorders, major depression, co-medication with benzodiazepines or other psychotropic medication during the past 4 weeks. Thus, in principle, the patients were drug free in order to minimize pharmacological influence, if possible. It should be noted that there was a high level of psychiatric comorbidity in the patient sample, as 23 of the 24 patients exhibited significant symptoms of other psychiatric disorders. The most common comorbid disorder $(\mathrm{n}=17)$ was dysthymia (F34.1). Depressive reaction (F43.2) was the next most common disorder $(n=4)$. There was also 1 case of an anxiety disorder (F41.1) and 1 case of neurasthenia (F48.0). The participants $(n=9)$ in the healthy control group were recruited from hospital employees and from the student population at the University of Munich. The 9 healthy volunteers were included following a medical examination (medical and psychiatric history) and the establishment of normal laboratory parameters (including normal values of haematological screening, blood chemistry with glucose, total protein, total bilirubin, liver enzymes, electrolytes, creatinine, urea, uric acid, cholesterol, triglycerides, semiquantitative urinalysis, and thyroid hormones, C-reactive protein, luteinizing hormone, folliculo-stimulating hormone, prolactin, progesterone, oestrogens, testosterone). They were in good general health and there was no evidence of a serious medical disease or a psychiatric disorder in any of the participants in the control group. As the controls were screened for psychiatric disorder prior to inclusion in the study, it was not considered necessary to conduct the same detailed psychological measurement that was conducted on the patients with SFD. In common with the (SFD) patient group, regular drug intake, the presence of acute or chronic infection and/or a recent history of an infectious disease within the past 4 weeks were the exclusion criteria. Female subjects were not taking birth control pills and had regular menstrual cycles, but the actual menstrual status was not considered. In both groups, IgE serum levels were determined in a certified routine laboratory. The study protocol was approved by the Ethical Committee of the University of Munich in accordance with the Declaration of Helsinki. Full written informed consent was obtained from each participant before study inclusion.

\section{Materials and Procedure}

Subjects fulfilling the inclusion criteria were invited to participate in the study and were assessed using a number of psychological measures. Blood serum levels of the different interleukins were also established for the 2 participant groups.

\section{Questionnaires and Measures}

The original version of TAS was developed by Taylor et al. [24] as a standardized self-assessment questionnaire to measure alexithymia. The authorized German version (TAS-26) has subsequently been developed by Kupfer et al. [20,21], which consists of 26 items that can be rated on a 5-point Likert scale and was used in our study to assess the presence and severity of alexithymia in the participants. This measure includes 26 items that generate scores on 3 dimensions: 'difficulty identifying feelings', 'difficulty describing feelings' and 'externally orientated thinking'. The
Table 1. Demographic and clinical characteristics of patients with SFD

\begin{tabular}{ll}
\hline Variable & $\begin{array}{c}\text { Patients with } \\
\text { SFD }(\mathrm{n}=24)\end{array}$ \\
\hline Age, years & $37.1(10.4)$ \\
Gender, male/female & $8 / 16$ \\
Somatization severity score (SOMS) & $16.3(10.5)$ \\
Global severity index (SCL-90-R) & $69.3(12.1)$ \\
HAMD & $13.4(4.2)$ \\
BDI & $18.8(9.6)$ \\
TAS-26 (T score) & \\
$\quad$ Total score & $55.7(8.2)$ \\
$\quad$ Factor 1 'difficulty identifying feelings' & $63.8(12.1)$ \\
$\quad$ Factor 2 'difficulty describing feelings' & $53.5(8.7)$ \\
Factor 3 'externally orientated thinking' & $47.4(12.6)$ \\
\hline
\end{tabular}

Figures in parentheses are SD.

German version was validated with a representative population sample $(\mathrm{n}=2,084)$ and shows adequate internal consistencies ranging between $r=0.67$ and $r=0.84$. Scores over raw value 54 (T score 61) on the TAS-26 are taken to indicate significant alexithymia, and this cut-off point is used to distinguish alexithymic and non-alexithymic individuals.

The SOMS [23] is a self-rating questionnaire by which 53 physical symptoms are investigated. The questionnaire includes all 33 physical complaints from the DSM-IV and the symptoms from ICD-10. Adding the number of positively answered symptoms allows computation of the 'somatization index' (ranging from 0 to 33 points), which was also used in our study. The number of selfrated somatization symptoms correlated with the number obtained by interview $(r=0.75)$, thus confirming the high validity of the SOMS.

The 90-item version of the Symptom Checklist-90 Revise (SCL-90-R) [25] is a widely used self-report questionnaire (90 items, 5-point Likert scale). The SCL-90-R asks for somatic and psychic complaints.

The 21-item Hamilton Rating Scale for Depression (HAMD) [26] was included in the present study to provide an observerrated measure of depression severity. This assessment was conducted by a fully trained psychiatrist (P.G.F). The 21-item Beck Depression Inventor (BDI) scale [27] was utilized in the present study to provide an index of self-rated depression severity.

\section{Assessment of Serum Levels of Interleukins}

Serum samples were centrifuged, and serum was frozen in aliquots at $-20^{\circ} \mathrm{C}$. Aliquots were defrosted, a standard curve of cytokines was established, and serum levels of the different interleukins were calculated (pg/ml) by using the Quantikine ${ }^{\circledR}(\mathrm{sIL}-2 \mathrm{R} \alpha)$ or the Quantikine high-sensitivity (IL-4, IL-6, IL-10, IL-12) enzyme-linked immunosorbent assay (ELISA) kit from R\&D Systems (Minneapolis, Minn., USA). Means of duplicate samples were used for calculation of the serum levels. The procedure was performed as recommended by the manufacturer. Infectious and inflammatory diseases, which could interfere with serum cyto- 
kine detection, were excluded by C-reactive protein, and the erythrocyte sedimentation rate was within normal limits in all patients. Furthermore, a hormone screen was performed to exclude endocrine diseases as cause of altered serum cytokine levels. There were 2 considerations when selecting the panel of cytokines investigated in the present study: first, we wanted to broadly cover the different immune functions, namely the Th1/Th2 dichotomy and the subsequent T/B cell activation that triggers cellular versus humoral immunity. IL-2 and IL- 6 reflect general immune activation, whereas IL- 4 is a cytokine of the Th2 immunity, which inhibits T effector cells and activates B cell immunity and herewith the humeral antibody response. IL-10 is produced by T cells and regulates Th2 differentiation. IL-12 is a member of the Th1 immunity that mediates T-cell-mediated effector mechanisms. Second, serum levels must be detectable in physically healthy humans. Interferon- $\gamma$ and tumor necrosis factor- $\alpha$ (Th1), IL-5 and IL-13 (Th2), and IL-1 $\alpha$ and IL-2 (generally proinflammatory) are not detectable in healthy volunteers. Although IL-4 is generally not detectable, we included this cytokine to refer to the publication by Corcos et al. [16]. sIL-2 R $\alpha$ was chosen instead of IL-2, as it has been shown in several investigations that sIL-2 R $\alpha$ correlates with increased $\mathrm{B}$ and $\mathrm{T}$ cell activation and immune system activation, e.g., rheumatoid arthritis or systemic lupus erythematosis [28].

\section{Data Analysis}

All data were analysed by SPSS for Windows@ 11.5. The data were examined for normal distribution using the Shapiro-Wilk test. Since most parameters were not normally distributed, nonparametric Mann-Whitney $U$ tests were performed to establish if any observed differences between the 2 groups in serum levels of the different interleukins were significant. Mann-Whitney U tests and Fisher's exact test were performed to test for significance of group differences for the parameters age, education and IgE. Interactions between the serum levels of the interleukins and participants' alexithymia (indexed by the participants' scores on the TAS-26) and depression scores (BDI, HAMD) were calculated with the non-parametric Spearman's coefficient. In order to elucidate the relationship between serum levels of the interleukins, participant characteristics and alexithymia, a forward stepwise multiple regression was conducted with serum levels (sIL-2 R $\alpha$, IL-4, IL-6, IL-10, IL-12), age and scores on BDI, HAMD, all SCL90-R scales entered as predictor variables, and participants' TAS26 scores entered as the dependent variable.

\section{Results}

\section{Participant Characteristics and Psychopathology}

Analysis of the participant characteristics (table 1) revealed that the age of the patients with SFD (mean 37.1 years, SD 10.4) did not differ significantly from the age of the controls (mean 32.2 years, SD 6.5; $\mathrm{p}=0.179$ ). A higher educational level was indicated in $45.8 \%$ of the patients and $55.6 \%$ of the controls $(p=0.123)$. Analysis of the participants' scores on the SOMS revealed that the patients with SFD exhibited elevated scores (mean 16.3, SD 10.5), indicating that these patients showed signs of moderate to severe somatization. A HAMD score of 13.4 (SD 4.2) demonstrated only moderate depression severity. Analysis of the participants' subjective general psychiatric symptomatology (indexed by the participants' scores on the SCL) revealed that the patients exhibited an elevated global severity index (mean 69.3, SD 12.1). Our patients exceeded the mean value (50) on all scales of SCL-90-R for somatization, obsessive compulsiveness, social insecurity, depression, anxiety, aggression, phobic anxiety, paranoid ideation, and psychoticism (data not shown).

\section{Assessment of Alexithymia}

Analysis of the participants' alexithymia (TAS-26) scores (table 1) revealed that the patients with SFD showed a TAS 'total' score (T score) of 55.7 (SD 8.2). Further inspection revealed that the patients scored significantly higher on the factor 1 of TAS ('difficulty identifying feelings') with 63.8 (SD 12.1) over the cut-off T score [20, 21]. However, the patients' scores on factor 2 of TAS ('difficulty describing feelings') with $53.5(\mathrm{SD}=8.7)$ and on factor 3 'externally orientated thinking' with 47.4 (SD 12.6) were not in the alexithymic range.

\section{Interleukins and Alexithymia}

Correlational analysis (table 2) revealed that sIL-2 R $\alpha$ was significantly negatively related to the patients' total TAS scores $\left[\mathrm{r}_{\mathrm{s}}(24)=-0.54, \mathrm{p}<0.05\right]$. This analysis also revealed a significant negative correlation between IL-4 serum levels and the patients' total TAS-26 scores $\left[r_{s}(24)=-0.49, p<0.05\right]$. There were no other significant relationships revealed by this analysis.

In order to further elucidate the nature of these relationships, a forward stepwise linear regression was conducted and revealed a significant model with sIL-2 R $\alpha$ entered as the only significant predictor of the patients' alexithymia scores $(\mathrm{p}=0.041)$ that accounted for $20 \%$ (16\%) of the variance in the patients' alexithymia (TAS26) scores $\left(\mathrm{R}^{2}=0.201, \mathrm{R}^{2}\right.$ adjusted $=0.159 ; \mathrm{F}_{1,20}=4.8$; $\mathrm{p}<0.05)$.

\section{Assessment of Interleukin Serum Levels}

Analysis of the serum levels of the different interleukins detected for the patients with SFD and healthy controls (table 3) revealed that the level of IL-10 was significantly augmented in the patients (mean $2.52 \mathrm{pg} / \mathrm{ml}$, SD 2.7 ) in comparison with the control group (mean 0.65 $\mathrm{pg} / \mathrm{ml}, \mathrm{SD} 1.3 ; \mathrm{U}=1.99$, with 24 patients and 9 controls; $\mathrm{p}<0.05)$. Furthermore, patients with SFD exhibited elevated levels of IL-6 (mean $2.61 \mathrm{pg} / \mathrm{ml}, \mathrm{SD} 1.4$ ) relative to 
Table 2. Relationships between alexithymia (TAS-26 scores) and serum levels of the different interleukins in the patients with SFD $(\mathrm{n}=24)$

\begin{tabular}{llllll}
\hline & \multicolumn{2}{l}{ Type of interleukin } & & \\
\cline { 2 - 6 } & sIL-2 $\alpha$ & IL-4 & IL-6 & IL-10 & IL-12 \\
\hline TAS, factor 1 'difficulty identifying feelings' & $\mathrm{r}_{\mathrm{s}}=-0.256$ & $\mathrm{r}_{\mathrm{s}}=0.025$ & $\mathrm{r}_{\mathrm{s}}=-0.204$ & $\mathrm{r}_{\mathrm{s}}=-0.083$ & $\mathrm{r}_{\mathrm{s}}=0.014$ \\
TAS, factor 2 'difficulty describing feelings' & $\mathrm{r}_{\mathrm{s}}=-0.306$ & $\mathrm{r}_{\mathrm{s}}=-0.071$ & $\mathrm{r}_{\mathrm{s}}=0.000$ & $\mathrm{r}_{\mathrm{s}}=0.133$ & $\mathrm{r}_{\mathrm{s}}=-0.088$ \\
TAS, factor 3 'externally orientated thinking' & $\mathrm{r}_{\mathrm{s}}=-0.027$ & $\mathrm{r}_{\mathrm{s}}=-0.248$ & $\mathrm{r}_{\mathrm{s}}=0.202$ & $\mathrm{r}_{\mathrm{s}}=-0.05$ & $\mathrm{r}_{\mathrm{s}}=0.346$ \\
TAS 'total' score & $\mathrm{r}_{\mathrm{s}}=-0.538^{*}$ & $\mathrm{r}_{\mathrm{s}}=-0.491^{*}$ & $\mathrm{r}_{\mathrm{s}}=0.162$ & $\mathrm{r}_{\mathrm{s}}=-0.031$ & $\mathrm{r}_{\mathrm{s}}=0.019$ \\
\hline
\end{tabular}

${ }^{*} \mathrm{p}<0.05$; correlations computed using Spearman's tests.

Table 3. Mean serum levels $(\mathrm{pg} / \mathrm{ml})$ of the different interleukins for SFD patients and healthy controls

\begin{tabular}{llll}
\hline $\begin{array}{l}\text { Type of } \\
\text { interleukin }\end{array}$ & $\begin{array}{l}\text { Patients with SFD } \\
(\mathrm{n}=24)\end{array}$ & $\begin{array}{l}\text { Healthy controls } \\
(\mathrm{n}=9)\end{array}$ & $\mathrm{p}$ value \\
\hline sIL-2 R $\alpha$ & $884.1(206.4)$ & $898.5(208.4)$ & 0.894 \\
IL-4 & $0.004(0.01)$ & $0.32(0.8)$ & 0.185 \\
IL-6 & $2.61(1.4)$ & $1.01(0.5)$ & $0.000^{*}$ \\
IL-10 & $2.52(2.7)$ & $0.65(1.3)$ & $0.047^{*}$ \\
IL-12 & $0.27(0.1)$ & $0.71(1.4)$ & 0.329 \\
\hline
\end{tabular}

Figures in parentheses are SD. Significance determined using Mann-Whitney $U$ tests with adjusted $\alpha$ for multiple comparisons; ${ }^{*} \mathrm{p}<0.05$.

the controls (mean $1.01 \mathrm{pg} / \mathrm{ml}$, SD 0.5; $\mathrm{U}=3.61$, with 24 patients and 9 controls; $\mathrm{p}<0.001$ ). On the other hand, the 2 groups did not differ significantly in terms of the serum levels of IL-4, IL-12 and sIL-2 R $\alpha$ (all tests $\mathrm{p}>0.05$ ). IL-4 levels were not detectable in the majority of probes, i.e. only in 7 patients and in 3 controls.

\section{Depression and Interleukins}

Inspection of the patients' depression scores only indicated moderate levels of depression, with 13.4 on the HAMD (SD 4.2) and 18.8 on the BDI (SD 9.6). Importantly, correlational analyses demonstrated no significant relationships between the patients' depression scores (HAMD and BDI) and the observed serum levels of the interleukins (data not shown).

\section{Immunoglobulin (IgE)}

Since the above described results suggested enhanced Th2 immunity in SFD patients, we determined the effector immunoglobulin in the serum. Accordingly, IgE serum levels were found to be significantly $(p=0.045)$ increased in SFD patients (mean $234 \mathrm{U} / \mathrm{ml}$, SD 278) in comparison with healthy controls (mean $53 \mathrm{U} / \mathrm{ml}$, SD 56.8).

\section{Discussion}

As expected, we found a higher prevalence of alexithymia in patients with SFD, regarding the TAS factor 1 'difficulty identifying feelings'. This is in agreement with empirical findings which support a positive association between somatization and alexithymia $[6,29]$. Consistent with our hypothesis, we found augmented IL- 6 and IL-10 in the patients with SFD relative to healthy controls. This pattern is interesting and suggests that the immune response in SFD is complex. IL-6 is a pro-inflammatory cytokine that is released in response to trauma or inflammation, and IL-10 is a regulatory cytokine that suppresses Th1 response and induces Th2 response in general immune activation [30]. However, these cytokines are not specific enough to discriminate between Th1 and Th2 predominance. Therefore, the significantly elevated $\operatorname{IgE}$ levels in our patients with SFD strongly support the hypothesis of an enhanced Th2-mediated immunity.

The finding of augmented IL- 6 is inconsistent with previous studies addressing immune response to SFD. For example, Rief et al. [12] reported significantly lower serum levels of IL- 6 in patients with somatization syndrome. It is plausible that the elevated IL-6 levels in the present study were a consequence of concurrent depression in our patients with SFD, because $87.5 \%$ exhibited comorbid depressive symptoms. Nevertheless, in previous studies [e.g., ref. 31, 32], higher IL-6 levels were found in depressed patients. However, in the present study, the lack of a significant correlation between self- or clinician- 
rated depression severity and serum levels of the interleukins does not fit with this proposal.

In contrast to our hypothesis, the observed changes in IL- 6 and IL-10 were not significantly related to the participants' alexithymia scores. However, it should be noted that serum levels of sIL-2 R $\alpha$ were negatively related to the patients' alexithymia scores; thus, high levels of alexithymia were associated with a reduction in the levels of these interleukins. Although the function of the sIL-2 R $\alpha$ is unclear, increased levels of the sIL-2 R $\alpha$ in biological fluids have been reported to correlate with increased immune system activation $[33,34]$. In our patients with SFD, serum levels of sIL-2 R $\alpha$ were negatively correlated with alexithymia. A plausible explanation of these results is that patients with more alexithymic features may be suffering from (unnoticed) chronic stress reaction with concomitant activation of Th2 response and an associated reduction in Th1-mediated immunity (indicated by the reduction in sIL-2 $\mathrm{R} \alpha$ ). This proposal is consistent with the hypothesis of Guilbaud et al. [19], who reported that alexithymic individuals may suffer from unnoticed chronic stress with associated changes in endocrine and immune functions, such that the Th1/Th2 balance is shifted towards Th2-dominant immunity (augmented IgE antibody synthesis), accompanied by a lowered cellmediated (Th1) immune response. The negative relationship between alexithymia and IL-4 serum levels, observed in the present study, is inconsistent with the findings of Corcos et al. [16], who reported a positive association in a group of 17 healthy females, between serum levels of IL- 4 and scores on the TAS factor 1 'difficulty identifying feelings'. However, in our study, IL-4 levels were not detectable in the majority of the patients, and therefore, the results must be interpreted with care despite the statistical significance. In comparison with Corcos et al. [16], who only included females, we investigated both genders in SFD in relation to alexithymia and interleukins. The effects are to be considered highly significant, without however regarding the gender effect in our study. It is valuable to examine the question of gender in further studies, because most studies in general population samples have found that men show more alexithymia than women $[35,36]$; however, other studies have observed no association between alexithymia and gender [37].

In summary, the findings of the present study are in agreement with the stress-alexithymia hypothesis [38], which posits that alexithymia is related to sympathetic hyperactivity. In line with this suggestion, Friedlander et al. [39] proposed that SFD may result from the alexithy- mic inability to differentiate and elaborate affect, which gives rise to physiological arousal and a negative subjective state. This decoupling between subjective and physiological arousal may increase the risk for alexithymic individuals of stress-related illnesses [40]. Furthermore, there is evidence that physical and psychological stressors can provoke transient increases in proinflammatory interleukins [41-43]. On the other hand, it is of interest that in recent research $[44,45]$, there have been indications that alexithymia might be associated with decreased basal activity of the hypothalamic-pituitary-adrenal axis. In contrast, in a recent study [46], a positive relationship between alexithymia and high cortisol levels was found. Other authors [47-49] even discussed hypocortisolism as a biological marker of stress-related disorders such as chronic fatigue syndrome or other idiopathic syndromes, like SFD. Thus, further studies have to focus on investigating neuroendocrine parameters in addition to immunology variables to clarify possible associations.

Some questions remain unanswered. For instance, increased serum markers of inflammation have been found in obese subjects [50,51], although the mechanism of action and the primary event are unclear. With respect to our study, obesity may strengthen an otherwise activated inflammatory process [52]. Alternatively, obesity may be the result of a psychological illness and may herewith influence immune responses. We have not assessed these parameters, but upcoming studies comparing alexithymic patients with healthy controls should include the evaluation of the body mass index and, if possible, the fat distribution pattern as well. Furthermore, although the healthy volunteers were routinely checked for any somatic disease or psychiatric disorder, they were not thoroughly assessed according to psychology measures and a structured clinical interview according to ICD-10, as the patient group had been. In addition, the control sample was relatively small, which may have influenced our ability to detect subtle changes in interleukin levels. However, most studies investigating immunological variables have used patients that have been treated with medication at the time of testing. This is important because increases in the circulating levels of tumor necrosis factor- $\alpha$, IL- 6 and other inflammatory cy tokines have been reported in response to psychotropic drugs, antipsychotics, antidepressants, and mood stabilizers [53-55]. Finding patients with SFD who are not currently medicated is difficult; thus, a major strength of our study was that none of our sample (patients and control group) were on medication at the time of testing. 
In summary, our study represents the first investigation of the relationship between alexithymic symptoms in SFD and changes of important cytokines. Further studies would be valuable to replicate our results and to evaluate the influence of immune balance in extended samples of patients with SFD and possibly later in therapeutic interventions.

\section{Acknowledgments}

We are grateful to Sabine Reinkunz, Roswitha Schneider, and Swetlana Paljan for their technical assistance.

\section{References}

1 Ustun TB, Sartorius N (eds): Mental Illness in General Health Care. Chichester, John Wiley, 1995.

2 Swartz M, Blazer D, Georg L, Landerman R: Somatization disorder in a community population. Am J Psychiatry 1986;143:14031408.

3 Escobar JI, Waitzkin H, Silver RC: Abridged somatization: a study in primary care. Psychosom Med 1998;60:466-472.

4 Barsky AJ, Orav E, Bates DW: Somatization increases medical utilization and costs independent of psychiatric and medical comorbidity. Arch Gen Psychiatry 2005;62:903910.

5 Bach M, Bach D: Predictive value of alexithymia: a prospective study in somatizing patients. Psychother Psychosom 1995;64: 43-48.

6 Bankier B, Aigner M, Bach M: Alexithymia in DSM-IV disorder. Comparative evaluation of somatoform disorder, panic disorder, obsessive-compulsive disorder, and depression. Psychosomatics 2001;42:235-240.

7 Sifneos PE: The prevalence of 'alexithymic' characteristics in psychosomatic patients. Psychother Psychosom 1973;22:255-262.

8 Naatanen P, Rynamen A, KeltikangasJarvinen L: The influence of alexithymia characteristics on the self-perception and facial expression of physiological stress state. Psychother Psychosom 1999;68:252-262.

9 Wise TN, Mann LS, Sheridan MJ: Relationship between alexithymia, dissociation and personality in psychiatric outpatients. Psychother Psychosom 2000;69:123-127.

10 Lundh LG, Simonsson-Sarnecki M: Alexithymia, emotion, and somatic complaints. J Pers 2001;69:483-510.

11 Minami M, Katayama T, Satoh M: Brain cytokines and chemokines: roles in ischemic injury and pain. J Pharmacol Sci 2006;100: 461-470.

12 Rief W, Pilger F, Ihle D, Bosmans E, Egyed B, Maes M: Immunological differences between patients with major depression and somatization syndrome. Psychiatry Res 2001;105:165-174.
13 Todarello O, Casamassima A, Marinaccio M, La Pesa MW, Caradonna L, Valentino L, Marinaccio L: Alexithymia, immunity and cervical intraepithelial neoplasia: a pilot study. Psychother Psychosom 1994;61:199204.

14 Todarello O, Casamassima A, Daniele S, Marinaccio M, Fanciullo F, Valentino L, Tedesco N, Wiesel S, Simone G, Marinaccio L: Alexithymia, immunity and cervical intraepithelial neoplasia: replication. Psychother Psychosom 1997;66:208-213.

15 Dewaraja R, Tanigawa T, Araki S, Nakata A, Kawamura N, Ago Y, Sasaki Y: Decreased cytotoxic lymphocyte counts in alexithymia. Psychother Psychosom 1997;66:83-86.

16 Corcos M, Guilbaud O, Paterniti S, Curt F, Hjalmarsson L, Moussa M, Chambry J, Loas G, Chaout G, Jeammet P: Correlation between serum levels of interleukin- 4 and alexithymia scores in healthy female subjects: preliminary findings. Psychoneuroendocrinology 2004;29:686-691.

17 Kelly-Welch A, Hanson EM, Keegan AD: Interleukin-4 (IL-4) pathway. Sci STKE 2005; 293:9.

18 Dantzer R: Somatization: a psychoneuroimmune perspective. Psychoneuroendocrinology 2005;30:947-952.

19 Guilbaud O, Corcos M, Hjalmarsson L, Loas G, Jeammet P: Is there a psychoneuroimmunological pathway between alexithymia and immunity? Immune and physiological correlates of alexithymia. Biomed Pharmacother 2003;57:292-295.

20 Kupfer J, Brosig B, Brahler E: Testing and validation of the 26-item Toronto Alexithymia Scale in a representative population sample. Z Psychosom Med Psychother 2000; 46:368.

21 Kupfer J, Brosig B, Brähler E: Toronto-Alexithymie-Skala-26, Deutsche Version. Göttingen, Hogrefe Verlag, 2001.

22 Hiller W, Zaudig M, Mombour W: International Diagnostic Checklists for ICD-10 and DSM-IV (Handbook). Seattle, Hogrefe \& Huber, 1996

23 Rief W, Hiller W, Heuser J: SOMS - Das Screening für Somatoforme Störungen. Manual zum Fragebogen. Bern, Hans Huber Verlag, 1997.
24 Taylor GJ, Bagby RM, Parker JDA: The Revised Toronto Alexithymia Scale: some reliability, validity, and normative data. Psychother Psychosom 1992;57:34-41.

25 Derogatis LR: SCL-90-R. Self-report symptom inventory; in Collegium Internationale Psychiatriae Scalarum (ed): Internationale Skalen für Psychiatrie. Weinheim, Beltz Verlag, 1994.

26 Hamilton M: A rating scale for depression. J Neurol Neurosurg Psychiatry 1960;23:5662.

27 Beck AT, Ward CH, Mendelson M, Mock F, Erbaugh F: An inventory for measuring depression. Arch Gen Psychiatry 1961;4:561571.

28 Wolf RE, Brelsford WG: Soluble interleukin2 -receptors in systemic lupus erythematosus. Arthritis Rheum 1988;31:729-735.

29 De Gucht V, Heiser W: Alexithymia and somatisation: quantitative review of the literature. J Psychosom Res 2003;54:425-434.

30 Kidd P: Th1/Th2 balance: the hypothesis, its limitations, and implications for health and disease. Altern Med Rev 2003;8:223-246.

31 Sluzewska A, Rybakowski J, Bosmans E, Sobieska M, Berghmans R, Maes M, Wiktorowicz $\mathrm{K}$ : Indicators of immune activation in major depression. Psychiatry Res 1996;64: 161-167.

32 Tsao CW, Lin YS, Chen CC, Bai CH, Wu SR: Cytokines and serotonin transporter in patients with major depression. Prog Neuropsychopharmacol Biol Psychiatry 2006;30: 899-905.

33 Waldmann TA: The IL-2/IL-2 receptor system: a target for rational immune intervention. Immunol Today 1993;14:264-270.

34 Fernandez-Botran R: Soluble cytokine receptors: their role in immunoregulation. FASEB J 1991;5:2567-2574.

35 Parker JDA, Taylor GJ, Bagby R: The 20-item Toronto Alexithymia Scale-III. Reliability and factorial validity in a community population. J Psychosom Res 2003;55:269-275.

36 Lane RD, Sechrest L, Riedel R: Sociodemographic correlates of alexithymia. Compr Psychiatry 1998;39:377-385. 
37 Loas G, Corcos M, Stephan P, Pellet J, Bizouard P, Venisse JL: Factorial structure of the 20-item Toronto Alexithymia Scale. Confirmatory factorial analyses in nonclinical and clinical samples. J Psychosom Res 2001;50: 255-261.

38 Martin JB, Pihl RO: Influence of alexithymic characteristics on physiological and subjective stress responses in normal individuals. Psychother Psychosom 45:66-77.

39 Friedlander L, Lumley MA, Farchione T, Doyal G: Testing the alexithymia hypothesis: physiological and subjective responses during relaxation and stress. J Nerv Ment Dis 1997; 185:233-239.

40 Stone LA, Nielson KA: Intact physiological response to arousal with impaired emotional recognition in alexithymia. Psychother Psychosom 2001;70:92-102.

41 DeRijk R, Michelson D, Karp B, Petrides J, Galliven E, Deuster P, Paciott, G, Gold PW, Sternberg EM: Exercise and circadian rhythm-induced variations in plasma cortisol differentially regulate interleukin-1 $\beta$ (IL$1 \beta)$, IL- 6 , and tumor necrosis factor- $\alpha$ (TNF$\alpha)$ production in humans: high sensitivity of TNF- $\alpha$ and resistance of IL-6. J Clin Endocrinol Metab 1997;82:2182-2192.

42 Zhou D, Kusnecov AW, Shurin MR, DePaoli M, Rabin BS: Exposure to physical and psychological stressors elevates plasma interleukin 6: relationship to the activation of hypothalamic-pituitary-adrenal axis. Endocrinology 1993;133:2523-2530.
43 Papanicolaou DA, Wilder RL, Manolagas SC, Chrousos GP: The pathophysiologic roles of interleukin- 6 in human disease. Ann Intern Med 1998;128:127-137.

44 Henry JP, Haviland MG, Cummings MA, Anderson DL, Nelson JC, MacMurray JP, McGhee WH, Hubbard RW: Shared neuroendocrine patterns of post-traumatic stress disorder and alexithymia. Psychosom Med 1992;54:407-415.

45 Spitzer C, Brandl S, Rose HJ, Nauck M, Freyberger $\mathrm{HJ}$ : Gender-specific association of alexithymia and norepinephrine/cortisol ratios. A preliminary report. J Psychosom Res 2005;59:73-76.

46 Finset A, Graugaard PK, Holgersen K: Salivary cortisol response after a medical interview: the impact of physician communication behaviour, depressed affect and alexithymia. Patient Educ Couns 2006;60: 115-124.

47 Alfvén G, de la Torre B, Uvnas-Moberg K: Depressed concentrations of oxytocin and cortisol in children with recurrent abdominal pain of non-organic origin. Acta Paediatr 1994;83:1076-1080.

48 Chrousos GP, Gold PW: A healthy body in a healthy mind - and vice versa - the damaging power of 'uncontrollable' stress. J Clin Endocrinol Metab 1998;83:1842-1845.

49 Ehlert U, Gaab J, Heinrichs M: Psychoneuroendocrinological contributions to the etiology of depression, posttraumatic stress disorder, and stress-related bodily disorders: the role of the hypothalamus-pituitary-adrenal axis. Biol Psychol 2001;57:141-152.
50 Yoshida T, Kaneshi T, Shimabukuro T, Sunagawa M, Ohta T: Serum C-reactive protein and its relation to cardiovascular risk factors and adipocytokines in Japanese children. J Clin Endocrinol Metab 2006;91:2133-2137.

51 Panagiotakos DB, Pitsavos C, Yannakoulia M, Chrysohoou C, Stefanadis C: The implication of obesity and central fat on markers of chronic inflammation: the ATTICA study. Atherosclerosis 2005; 183:308-315.

52 Oeser A, Chung CP, Asanuma Y, Avalos I, Stein CM: Obesity is an independent contributor to functional capacity and inflammation in systemic lupus erythematosus. Arthritis Rheum 2005;52:3651-3659.

53 Pollmächer T, Haack M, Schuld A, Kraus T, Hinze-Selch D: Effects of antipsychotic drugs on cytokine networks. J Psychiatr Res 2000;34:369-382.

54 Hinze-Selch D, Schuld A, Kraus T, Kühn M, Uhr M, Haack M, Pollmächer T: Effects of antidepressants on weight and on the plasma levels of leptin, TNF- $\alpha$ and soluble TNF receptors: a longitudinal study in patients treated with amitriptyline or paroxetine. Neuropsychopharmacology 2000;23:13-19.

55 Haack M, Hinze-Selch D, Fenzel T, Kraus T, Kühn M, Schuld A, Pollmächer T: Plasma levels of cytokines and soluble cytokine receptors in psychiatric patients upon hospital admission: effects of confounding factors and diagnosis. J Psychiatr Res 1999;33:407418 